ures only $1.5 \mathrm{~mm}$ thickness and $8 \mathrm{~mm}$ in diameter, and connected with FM transmitter.

Several instances of experimental as well as clinical use are to be presented.

\title{
C-7. The Treatment of Acute Brain Swelling After Release of Intracranial Hypertension
}

\author{
-The Differential Effects of Cerebral Circulation \\ and Metabolism-
}

\author{
Nari-uki Hayasui, Hisao Tamura, Hideo Inoue, Takiji Shugawara, \\ Takashi Tsubokawa and Nobuo Moriyasu \\ Department of Neurosurgery, Nihon University, School of Medicine
}

The effects of increased intracranial pressure (ICP) upon the hemodynamicsresponses and total cerebral blood flow have been well defined. But little knowledge is known about the changes of regional cerebral blood flow (rCBF) and blood, csf acid-base balance following release of intracranial hypertension (ICH).

In order to get the information about correct therapy the present study was designed to musurements fo the alteration of $\mathrm{rCBF}$, ICP, sagital venous pressure, blood acid-base balance and vital signs (simultaneously) during and after release of increase of ICP. The cortical blood vessels were observed through a cranial window with a microscope $(\times 20)$. The effects of dehydration, hyperventilation, hemodylusion therapy, central depressor medication, administration of THAM-sol. and the combination of these therapies were studied on the acute brain swelling occuring after release of $\mathrm{ICH}$.

The results are summarized as follows;

1) Progressive arterial hypoxemia is induced following intracranial hypertension in all cases, but acid-base balance is altered depending on the grade of height of ICP. According to the relationship the grade of ICP and the alteration of the acid-base balance 4 stages are identified as follows; stage 1: normally-slightly metabolic acidosis, stage 2 : hypoxic respiratory alkalosis; stage 3 : severe metabolic acidosis; stage 4: metabolic and respiratory acidosis.

2) During increased intracranial pressure by compression, there is marked decrease of $\mathrm{rCBF}$ at the compressed area, but the clearance is distinctly accerated except subcortical $\mathrm{rCBF}$ at the surrounding area of compression. Following release of moderate brain compression by balloon, $\mathrm{rCBF}$ at both area increased markedly without any change of perfusion pressure (Mean Arterial Blood Pressure-Sagital Sinus Wedge Pressure).

3) Circulatory disturbance is most severe in the venous side and perfusion pressure gradually decrease and finally cease in capillaries and later in the lager vessels associated with high grade of $\mathrm{ICH}$. The causes of reduction and cessation 
of circulation are not only external compression on the vessels, but also the alteration vessels themselves, such as internal occlusion by the sludged cells, microemboli and intravascular clotting in vessels.

4) Following the deflation of the balloon, acute brain swelling (stage 2-4) was observed associated with the alteration of cerebral circulation in the majority of the capillaries and small vessels. Microcirculatory disturbances caused by intravascular occlusion must be considered as a cause of acute brain swelling following release of $\mathrm{ICH}$.

According to these experimental results, the treatment of acute brain swelling after release of intracranial hypertension, both the dehydration and hyperventilation is not effective therapy, but the combination therapy of hemodylusion and hyperventilation or administration of THAM solation would be usefull therapy for such severe acute brain swelling following release of $\mathrm{ICH}$.

The useful clinical indicators selecting correct therapy for the cases, are increasing of intracranial arterio-venous $\mathrm{O}_{1}$ defference in the first choice.

\title{
C-8. Acute Brain Swelling
}

\section{Chemical Factor and Cerebrovascular Tone-}

Hiroyu Ota, Kiyoshi Imatsuki, Katsujiro Matsumoto,

Syogo Nagao and Akira Nishimoto

Department of Neurosurgery, University of Okayama

\section{C-9. Formation and Absorption of Cerebrospinal Fluid in Increased Intracranial Pressure}

\author{
Youichi Irsaka, Shigeo Toya, Hisao Shizawa, Taizo Tsukumo, \\ Kinya Oshida, Toru Nakanishi and Morikazu Ueda \\ School of Medicine, Keio University
}

The technique of cerebrospinal fluid perfusion by Pappenheimer has made a great progress in understanding the CSF dynamics.

Fifteen dogs were perfused from ventricle to cisterna magna with artificial CSF containing $10 \mathrm{uCi}$ of 111I-RISA in $500 \mathrm{ml}$.

The successive $10 \mathrm{~min}$ samples were collected and measurements were made on inflow rate, outflow rate and radioactivity.

The CSF formation rate was increased in accordance with the elevation of the 